\title{
THE STRUCTURE OF REGULATED FUNCTIONS
}

\author{
CASPER GOFFMAN, GADI MORAN AND DANIEL WATERMAN
}

\begin{abstract}
It is shown that there is a nontrivial class of regulated functions each of which is a representable as the sum of a continuous function and a uniformly convergent series of jump functions whose jumps are those of the given function. The set of regulated functions is the union of the classes of functions of bounded $\Phi$-variation for convex $\Phi$.
\end{abstract}

The regulated functions on a closed interval are those functions whose right and left limits exist at each point. Every regulated function is bounded, has a countable set of discontinuities, and is the limit of a uniformly convergent sequence of step functions. The regulated functions are of importance in the theory of stochastic processes and in the theory of everywhere convergence of Fourier series.

Functions of bounded variation are regulated, as are the functions of bounded $\Phi$-variation [1] and the functions of bounded $\Lambda$-variation [3]. In $\S 1$ we shall show that each regulated function is of bounded $\Phi$-variation for some $\Phi$.

A function of bounded variation has a canonical representation as the sum of a continuous function of bounded variation and a sum of jump functions, the jumps being those of the given function of bounded variation. In $\$ 2$ we shall investigate the possibility that a regulated function have a representation as the sum of a continuous function and a uniformly convergent series of jump functions whose jumps are those of the given function. We shall see that, although we can characterize a nontrivial class of functions for which this representation is possible, we can construct functions which have no such representation.

1. Let $\Phi(x)$ be a convex function on $[0, \infty)$ with $\Phi(0)=0, \Phi(x)>0$ for $x>0$. A function $f$ defined on an interval $I$ is said to be of bounded $\Phi$ variation $(\Phi-B V)$ if the $\Phi$-variation of $f$,

$$
V_{\Phi}(f)=\sup \left\{\sum \Phi\left(\left|f\left(I_{n}\right)\right|\right):\left\{I_{n}\right\}\right\}<\infty,
$$

where $\left\{I_{n}\right\}$ denotes a partition of $I$ into intervals $\left[a_{n}, b_{n}\right]$ and $f\left(I_{n}\right)=f\left(b_{n}\right)$ $-f\left(a_{n}\right)$. It is usual to consider only those $\Phi$ for which $\Phi(x) / x \rightarrow 0$ as $x \rightarrow 0$ in order that $\Phi-B V$ should contain $B V$ properly.

It is clear that every function of $\Phi-B V$ is regulated. We shall suppose that at each point of discontinuity of a regulated function $f$,

Received by the editors April 18, 1975.

AMS (MOS) subject classifications (1970). Primary 26A15. 


$$
\min \{f(x+), f(x-)\} \leqslant f(x) \leqslant \max \{f(x+), f(x-)\} .
$$

We shall show that the class of regulated functions is the union of the classes $\Phi-B V$. An analogous theorem for functions of bounded $\Lambda$-variation has been obtained by S. J. Perlman [2].

THEOREM 1. If $f$ is regulated, then $f$ is of bounded $\Phi$-variation for some $\Phi$ with $\Phi(x) / x \rightarrow 0$ as $x \rightarrow 0$.

Proof. Let the domain of $f$ be $[0,1]$. We suppose, at first, that $f$ is continuous. Let $\omega$ be the modulus of continuity of $f$ and choose $k_{n} \searrow 0, n$ $=1,2, \ldots$, such that

$$
\sum n k_{n}<\infty \text { and } k_{n} / \omega(1 / n) \rightarrow 0 .
$$

Let $\Phi$ by any convex function on $[0, \infty)$ whose graph on $[0, \omega(1)]$ coincides with the lower boundary of the convex hull of $\left\{\left(\omega(1 / n), k_{n}\right): n=1,2, \ldots\right\}$. Then

$$
\sum n \Phi(\omega(1 / n))<\infty \text { and } \Phi(x) / x \rightarrow 0 \text { as } x \rightarrow \infty .
$$

If $\left\{I_{k}\right\}$ is a partition of $[0,1]$, then

$$
\sum \Phi\left(\left|f\left(I_{k}\right)\right|\right) \leqslant \sum n \Phi(\omega(1 / n))<\infty,
$$

implying that $f \in \Phi-B V$.

Supposing now that $f$ is regulated on $[0,1]$, let $\left\{a_{n}\right\}$ be the points of discontinuity of $f$. Let

$$
y(x)=x+\sum_{n: a_{n}<x} 2^{-n}, \quad x \notin\left\{a_{n}\right\},
$$

and

$$
F(y(x))=f(x), \quad x \notin\left\{a_{n}\right\} .
$$

Let

$$
t_{n}^{-}=\lim _{x>a_{n}} y(x), \quad t_{n}^{+}=\lim _{x \searrow a_{n}} y(x) .
$$

On each $\left[t_{n}^{-}, t_{n}^{+}\right]$, define $F(t)$ to be the linear function whose graph is the segment connecting $\left(t_{n}^{-}, f\left(a_{n}-\right)\right)$ and $\left(t_{n}^{+}, f\left(a_{n}+\right)\right)$. Then $G(t)=F(t / 2)$ is continuous on $[0,1]$ and there is a $\Phi$, as above, such that $G \in \Phi-B V$. For any partition $\left\{I_{n}\right\}$ of $[0,1]$, there exists a collection of nonoverlapping intervals $J_{n} \subset[0,1]$ such that

$$
\sum \Phi\left(\left|f\left(I_{n}\right)\right|\right)=\sum \Phi\left(\left|G\left(J_{n}\right)\right|\right) \leqslant V_{\Phi}(G)<\infty,
$$

and so $f \in \Phi-B V$.

2. Let $f$ be a regulated function on $[a, b]$. We assume now that $f$ is left continuous everywhere and continuous at $b$. Let the points of discontinuity of $f$ be $\left\{a_{n}\right\}, n=1,2, \ldots$, and the saltus of $f$ at $a_{n}$ is then $k_{n}=f\left(a_{n}+\right)-f\left(a_{n}\right)$. Then, although $\lim k_{n}=0$, the series $\sum k_{n}$ need not converge. A jump function $J_{c}^{k}$ is defined by 


$$
J_{c}^{k}(x)=0, \quad x \leqslant c \text { and } J_{c}^{k}(x)=k, \quad x>c .
$$

Our problem is to decide for which regulated $f$ with saltus $k_{n}$ at $a_{n}, n=1$, $2, \ldots$, there is a rearrangement $\left\{a_{n_{i}}\right\}$ of $\left\{a_{n}\right\}$ and a continuous function $g$ such that

$$
f=g+\sum_{i=1}^{\infty} J_{a_{n_{i}}}^{k_{n_{1}}}
$$

the series being uniformly convergent.

We define the saltus function $f^{*}$ of $f$ by

$$
f^{*}(x)=f(x+)-f(x-) .
$$

Then $f^{*}$ is zero except on a countable set. For each $\varepsilon>0, \quad\left\{x:\left|f^{*}(x)\right|>\varepsilon\right\}$ is finite. We shall first show that this is the essential property of the saltus function of a regulated function.

THEOREM 2. If $h$ is a function on $[a, b]$ such that $\{x:|h(x)|>1 / n\}$ is finite for $n=1,2, \ldots$, and $h(a)=h(b)=0$, then there is a regulated function $f$ such that $f^{*}=h$.

Proof. Without loss of generality we may assume $\max |h(x)| \leqslant 1$. For a given positive integer $n$, let the finite set $\left\{x: 2^{-n}<|h(x)| \leqslant 1\right\}=\left\{x_{i}\right\}, i=1$, $\ldots, m$, where $x_{i}<x_{i+1}$. Set $x_{m+1}=b$. Let $k_{i}$ be the integer such that $2^{-k_{i}-1}<\left|h\left(x_{i}\right)\right| \leqslant 2^{-k_{i}}$.

Now let $f_{n}(x) \equiv 0$ for $x \in\left[a, x_{1}\right], f_{n}\left(x_{i}\right)=0$ for $i=1, \ldots, m+1$, and let $f_{n}(x)$ be linear on $\left(x_{i}, x_{i+1}\right], i=1, \ldots, m$, with $f_{n}\left(x_{i}+\right)=h\left(x_{i}\right) \cdot 2^{-\left(n-k_{i}\right)}$.

It is easily seen that $\sum f_{n}(x)$ converges uniformly to a regulated function $f$ such that $f^{*}=h$.

In the sequel "an interval" means "an interval with more than one point", and $I$ denotes a subinterval of $[a, b]$. For $h: I \rightarrow R$ let $h_{+}(x)=\max \{h(x), 0\}$, $h_{-}(x)=\max \{-h(x), 0\}$. The sums $\sum_{x \in I} h_{+}(x), \sum_{x \in I} h_{-}(x)$ may be finite or infinite.

If both are infinite we shall way that $f$ is of type $(1,1)$ on $I$, if both are finite we shall say that $f$ is of type $(0,0)$ on $I$, if the first is infinite and the second finite we shall say that $f$ is of type $(1,0)$ on $I$, and if the first is finite and the second infinite we shall say that $f$ is of type $(0,1)$ on $I$.

Clearly, if $f$ is of type $(1,0)$ or $(0,1)$ on a subinterval then $f$ has no representation (*). We shall show that if a regulated function is of type $(1,1)$ on every interval, then it has a representation (*). First we shall show that there exist regulated functions which are of type $(1,1)$ on every interval and that there exist regulated functions which do not have a representation (*), but are of type $(1,1)$ or $(0,0)$ on each interval.

EXAMPLE 1. Regulated functions which are of type $(1,1)$ on each subinterval $J$ of $[0,1]$.

By Theorem 2, we need only find an $h:[0,1] \rightarrow R$ satisfying the conditions of that theorem such that

$$
\sum_{x \in J} h_{+}(x)=\sum_{x \in J} h_{-}(x)=\infty \text { for every subinterval } J \text { of }[0,1] .
$$




$$
h\left(\frac{2 p+1}{2^{n}}\right)=\frac{(-1)^{n}}{n}, \quad p=0, \ldots, 2^{n-1}-1 ; n=1,2, \ldots
$$

and $h(x)=0$ otherwise.

EXAMPLE 2. A regulated function which does not have a representation (*) and is of type $(0,0)$ or type $(1,1)$ on every interval.

Let $\left\{I_{n}\right\}$ be a sequence of nonoverlapping intervals with $I_{n}$ to the left of $I_{n+1}$ and $\cup I_{n}=[0,1)$. On $I_{2 k-1}, \quad k=1,2, \ldots$, let $h(x)=1 / k$ at $k^{2}$ interior points and be zero elsewhere. On $I_{2 k}, \quad k=1,2, \ldots$, define a function $\bar{h}(x)$ in a manner analogous to the construction in Example 1 and then set $h(x)=\bar{h}(x) / k$. Then from Theorem 2 , there exists a regulated $f$ such that $f^{*}=h$. On any interval which intersects the interior of an $I_{2 k}, f$ is of type $(1,1)$; on other intervals it is of type $(0,0)$. If $f$ had the representation $(*)$, then the sum of the jumps would be bounded, but this sum has increment $k$ on $I_{2 k-1}$.

We turn now to our final result.

THEOREM 3. If $f$ is regulated on $[a, b]$ and is of type $(1,1)$ on every subinterval, then $f$ has a representation (*).

Proof. Suppose $f$ is of type $(1,1)$ on every subinterval of $[a, b]$ and $M=\max \left|f^{*}(x)\right|$. Let $S=\left\{x: f^{*}(x) \neq 0\right\}$. We begin by showing that given $\varepsilon>0$ and $\eta>0$ there is a finite set $F \subset S, F=\left\{\alpha_{1}, \ldots, \alpha_{r}\right\}$, where $\alpha_{1}$ $<\cdots<\alpha_{r}$, such that

$$
\sum_{x \in F} J_{x}^{f^{*}(x)}=\sum_{i=1}^{r} J_{\alpha_{1}}^{f^{*}\left(\alpha_{i}\right)}
$$

satisfies

$$
\left|\sum_{i=1}^{k} J_{\alpha}^{f^{*}\left(\alpha_{i}\right)}(x)\right| \leqslant M+\varepsilon \text { for } 1 \leqslant k \leqslant r, x \in[a, b],
$$

and

$$
\max \left\{\left|f^{*}(x)\right|: x \in S \backslash F\right\}<\eta .
$$

To show this, let $F_{1}=\left\{a_{1}, \ldots, a_{s}\right\}$, where $a_{i} \in S, a<a_{1}<\cdots<a_{s}$ $<b$ and $\max \left\{\left|f^{*}(x)\right|: x \in S \backslash F_{1}\right\}<\eta$. For each $i=1, \ldots, s-1$, choose $\left\{a_{i j}\right\}$, a finite subset of $S, a_{i}<a_{11}<\cdots<a_{i n_{i}}<a_{i+1}$, such that the sign of $f^{*}$ at each $a_{i j}$ is opposite to its sign at $a_{i}$ and $\left|f^{*}\left(a_{i}\right)+\sum_{j=1}^{n_{i}} f^{*}\left(a_{i j}\right)\right|<\varepsilon / S$. Let $F$ be the set $a_{1}<a_{11}<\cdots<a_{1 n_{1}}<a_{2}<a_{21}<\cdots<a_{s-1}<\cdots$ $<a_{s}$. The function $\sum_{x \in F} J_{x}^{f^{*}(x)}$ has property (i) and $F$ has property (ii).

We now show that a regulated function $f$ of type $(1,1)$ on every subinterval has a representation (*). We assign indices to the elements of $S$ in the course of our construction, rather than rearranging a given index set.

Let $\left\{\varepsilon_{n}\right\}$ be a sequence of positive numbers such that $\sum \varepsilon_{n}<\infty$. Let $T_{1}=\left\{x:\left|f^{*}(x)\right| \geqslant 2^{-1}\right\}$ and, for $n=2,3, \ldots$, let $T_{n}=\left\{x: 2^{-n-1} \leqslant\left|f^{*}(x)\right|\right.$ $\left.<2^{-n}\right\}$. Applying the above remark to $f$ with $\varepsilon=\varepsilon_{1}$ and $\eta=2^{-1}$, we obtain an ordered set $S_{1}=\left\{a_{i}\right\}, i=1, \ldots, i_{1}$, with $a_{i}<a_{j}$ for $i<j$ and $S_{1} \supset T_{1}$. Let 


$$
f_{1}=f-\sum_{x \in S_{1}} J_{x}^{f^{*}(x)} .
$$

Now $\max \left|f_{1}^{*}(x)\right|<2^{-1}$. Applying the above remark to $f_{1}$ with $\varepsilon=\varepsilon_{2}$ and $\eta$ $=2^{-2}$, we obtain set $S_{2}=\left\{a_{i}\right\}, i=i_{1}+1, \ldots, i_{2}$, with $a_{i}<a_{j}$ for $i<j$, $S_{1} \cap S_{2}=\varnothing$, and $S_{1} \cup S_{2} \supset T_{1} \cup T_{2}$. Let

$$
f_{2}=f_{1}-\sum_{x \in S_{2}} J_{x}^{f^{*}(x)}
$$

Having obtained $f_{n}$, where $n$ is a positive integer and $\max \left|f_{n}^{*}(x)\right|<2^{-n}$, and a finite set $S_{n}$ with $S_{n} \cap S_{j}=\varnothing, j<n$, and $S_{1} \cup \cdots \cup S_{n} \supset T_{1} \cup \cdots$ $\cup T_{n}$, we apply the above remark to $f_{n}$ with $\varepsilon=\varepsilon_{n+1}$ and $\eta=2^{-(n+1)}$ to obtain the set $S_{n+1}=a_{i_{n}+1}, \ldots, a_{i_{n+1}}$, with $a_{i}<a_{j}$ for $i<j, S_{n+1} \cap\left(\cup_{1}^{n} S_{j}\right)$ $=\varnothing, S_{n+1} \supset T_{n+1}$. Let

$$
f_{n+1}=f_{n}-\sum_{x \in S_{n+1}} J_{x}^{f^{*}(x)}=f-\sum_{i=1}^{i_{n+1}} J_{a_{i}}^{f^{*}\left(a_{i}\right)} .
$$

The saltus of $f_{n}$ at $x$ is less than $2^{-n}$ for each $x \in[a, b]$ and the sequence $\left\{f_{n}\right\}$ converges uniformly to a function $g$ which is, therefore, continuous. Then

$$
f=g+\sum_{i=1}^{\infty} J_{a_{i}}^{f^{*}\left(a_{i}\right)}
$$

is the desired representation.

\section{REFERENCES}

1. J. Musielak and W. Orlicz, On generalized variations. I, Studia Math. 18 (1959), 11-41. MR 21 \#3524.

2. S. Perlman, Functions of generalized variation, Rev. Roumaine Math. Pures Appl. (to appear).

3. D. Waterman, On convergence of Fourier series of functions of generalized bounded variation, Studia Math. 44 (1972), 107-117. MR 46 \#9623.

DEPARTMENT OF MATHEMATICS, PURDUE UNIVERSITY, WEST LAFAYETTE, INDIANA 47907 (Current address of C. Goffman and G. Moran)

DEPARTMENT OF MATHEMATICS, SYRACUSE UNIVERSITY, SYRACUSE, NEW YORK 13210 (Current address of D. Waterman) 